\title{
IMPROVING CHANGE MANAGEMENT: HOW COMMUNICATION NATURE INFLUENCES RESISTANCE TO CHANGE
}

\author{
Paula Matos Marques Simoes \\ Fundacao Dom Cabra\& Grenoble Ecole de Management \\ Mark Esposito \\ Grenoble Ecole de Management \& University of Cambridge, CPSL
}

\begin{abstract}
Little has been studied yet in terms of how communication nature influenceschange process. This article explores a case studythat takes partin a broaderresearch project, aimed to contribute in

this direction. It was applied a mix method approach to characterize resistance to change and

communication nature within one organization under a radical change process. One main theoreticalcontribution is an instrumental grid to characterize dialogic communication nature.Findings of the case study indicate that resistance to change reduces under dialogic communication and by revealing how communication dimensions perform in time,practitioners may enhance guidelines to effective change communication management.
\end{abstract}

It's already a common sense that changes are no longer happening sparsely, but are the pattern for nowadays. As well as widely announced scenarios of broader, faster changes (Eisenhardt 1989; Burnes and James 1995) organizations are immersed in andthe high levels of uncertainty these contemporary challenges promote. Such is especially true when change impacts many units in an organization, thus amplifying the number of people and perspectives involved, and leading to higher business complexity, as Buono and Keeneth (2008) have identified. One could argue that changes have always had been the pattern, and we, as observers, are the ones that are more aware and conscious about it than before. Anyway, this perception of organizational environment is requiring constant development of management science to deal with change processes.

Impact and frequency of change efforts are the most common classification, although not extensive criteria, to characterize organizational change. As a complex phenomenon, it can be examined from several dimensions. A possible organization of 
the theme by ontological assumptions is offered by Palmer and Dunford (2008). And due to business complexity and social-technical uncertainty arguments for selecting an approach, it can be advocated the appropriateness of adopting a constructionist/organicist - as an alternative to an instrumentalist/mechanistic interpretation of organizational change.

Furthermore, as faster and faster changes take place it is expected that overlapping efforts will occur, and in such situations the outcomes are only partially anticipated, as many interactions and interests will have to be accommodated. Actually, results would be only partially predicted as, with the intentionality of actions and discussions, proposed outcomes and several forces influencing it in different directions would coexist. Accordingly, the Sensemaking Theory of Change would be applicable lens for contemporary changes in organizations.

\section{$\underline{\text { Communication }}$}

Communication has been recognized as a relevant dimension to the success of organizational change, and it is considered important in building change readiness, reducing uncertainty, and as a key factor in gaining commitment (Armenakis, Harris et al., 1993; Klein, 1994). The design and conduct of strategy communication in change processes require decisions that integrate different perspectives of analysis and a relative effort, but that may be important to minimize the resistance to change (Argyris, 1994).

According to Caldwell (1993), communication within the context of change has been seen as a way to inform, involve and even motivate collaborators to participate in the change process wholeheartedly. Such approach reveals communication as a technique and a tool (Reis, 2004.).

Alternatively, by recovering the etymological root of the word "communication" one can find the Latin word "communicatione" meaning to participate, to pool or to take common action. More closely aligned to this original connotation, communication can be taken as a social process where people, immersed in a particular culture, create and exchange meanings, thus addressing the reality of everyday experience (Gill and 
Adams, 1998:41, cited by Souza (2006). Communication, therefore, is not the transmission of meanings but the joint construction of meaning. This definition implies that messages exchanged only have cognitive effects and create meaning because they are assigned meanings, and that such meanings depend on the general culture and context in which it occurs. Communication then, is by no means limited to verbal productions or even written expressions. Its range is larger, and it includes gestures, actions and behaviors in general.

A continuous exchange of messages can allow new meanings to develop, and these will stem from changes in each individual's original meanings, which can lead to a process of meaning convergence that can be understood as the beginning of communication, the possession of something in common. It is worthwhile to alert, on the other hand, that a broad provision of initiatives and communication tools do not necessarily lead to successful change, in terms of intended results. That is, it is not only the quantity but mainly the quality, or as Reis (2002) warns, the nature of the communicative mediation implemented that can influence change. Here there is a distinction in the nature of communication strategies. Those whose role or function is that of justifying and rationalizing change will make use of traditional communication techniques that are usually used to systematize, disseminate and express information. In such cases, change is communicated to the staff (Caldwell, 1993) and, even when the means and messages are intensified in number and sophistication while various resources are invested, they may not lead to reduced resistance to change due to their not privileging the building of shared meanings.

Conversely, communication strategies whose role or function is to refine and align change can be more successful at reducing individual resistance to change, as they allow the meaning attributed to change to be constantly re-elaborated by means of cyclical contributions within the cognitive process of its interlocutors. To put it another way, they can produce two-way communicational structures by encouraging learning through mistake and dialogic interaction. They incorporate, structurally or within their dynamics, the possibility of participation, of inclusiveness (Reis, 2004.), or even, as 
Lewis (2007) advocates, they can be better configured as an input focus than as a dissemination focus. Aligned with that, Elving (2005) stated that communication "aims for the creation of mutual understanding and trusting relationships".

The connection between communications and organizational change has been attracting increasing interest from scholars and practitioners during the last decade (Johansson and Heide, 2008). Several of them have emphasized the important role of communications in change processes (Ford and Ford, 1995; Kotter, 1996; Lewis and Seibold, 1996; Daly, Eague et al., 2003; Elving, 2005), and according to some of them, communications and organizational change are inextricably linked processes (Lewis, 1999). It can be easily found, throughout the literature review, that change is a communicative challenge (Allen et al 2007), or in another similar view, that change implementation is primarily a communication problem (Lewis and Seibold, 1998). As Bordia et al (2004: 96) illustrate:"Communication processes are inherently a part of these implementation activities".

Thus, it is necessary to look deeper into relational and communicational processes while recognizing that sensemaking and sensegiving, and consequently change itself, are not under complete control. As the sensemaking theory of change and the other socially-constructed perspectives suggest, all that is involved in change will also bring about change through their social processes of interaction and the meanings they develop as a result (Berger and Luckmann, 1976). Indeed, such a perspective will shed light on some dialectical distinctions, revealing those that would not be the best denomination. As for now, change recipients might be better denominated change participants, due to the active, and not passive, role they play in change (Balogun and Johnson, 2004). As discussed further in this literature review, this "redenomination" of roles would also be applied to the resistant to change label.

As an influenced but not controlled process, somehow sensemaking can generate disparate senses in comparison to what was expected from change leaders. If sensemaking occurs in the direction expected, or if comes up with an unexpected response, this is essential information that needs to be monitored by change leaders. 
Responsiveness to change, or resistance to change as it has been usually called, has also been a deeply studied aspect of organizational change that will be advanced in the following section in both conceptual and operational dimensions.

\section{Resistance to Change}

Resistance to change has usually been recognized as a significant factor that can influence the outcomes of an organizational change effort (Chiung-Hui and IngChung, 2009). Organizational change literature offers several definitions for resistance to change. In fact, the common point among most of these definitions is that resistance to change is seen as an important reason for change process failures (Armenakis, Harris et al., 1993), and consequently, as a problem to be minimized or overcome (Nadler, 1993). One can find an example of this perspective in Coetsee's statement, as resistance to change can be understood as "opposed or blocking energies and power directed at impeding, redirecting, rejecting or stopping change" (Coetsee, 1999:209).

When the concept of resistance to change is re-elaborated, it is directed to an extensive alteration towards a communicative aspect of the change process:

"Resistance, properly understood as feedback, can be an important resource in improving the quality and clarity of the objectives and strategies at the heart of a change proposal. And, properly used, it can enhance the prospects for successful implementation". (Ford and Ford, 2009: 103)

This approach is supported by other scholars. According to Schein (2003), dealing with defensive routines requires supplying a space for reflection and dialogue. Moreover, Ford, Ford et al (2008) reflects that when much responsible insight about resistance is present, one that considers it as a result of an agent's own actions and sensemaking, the agent can purposely opt to make sense of recipient expressions as a 
counter offer. Therefore, in this case the role of change agent definitely includes taking charge of the change dialogues in order to overcome apparently resistive behaviors "by bringing both agent and recipient background conversations to the fore" (2008:373). A truly provocative idea is that expressing opposition can be, in fact, a sign of deep commitment to change by being brave enough to show concern about obstacles not yet seen(Stohl and Cheney, 2001: 380).

Due to this more comprehensive point of view on resistance to change, it is necessary to recognize that the term should be altered purposively as a response to change, echoing Dent and Golberg (1999) and Oreg's (2006) claims that the expression resistance to change is limiting and not adequate. In this literature review, such alteration emphasized the nature of subjective experiences in order to obtain a more valid understanding of what resistance is actually about. On the other hand, as the predominant term used in the literature provided on the empirical studies of the phenomena is resistance to change, it will be useful to adopt the same term and construct as the basis for operational application.

Taking the point of view of the Sensemaking Theory of Change as a basis, resistance to change is better defined, from an operational point of view, according to Piderit (2000), as a tridimensional attitude towards change, which includes behavioral, affective and cognitive components. Naturally, there is some interdependence among the three components, as what change agents and participants feel about a change will often correspond to what they think about the change and to their behavioral intentions in its regard. Nevertheless, the components are distinct, and each one highlights a different aspect of the resistance structure.

One of the latest and most renowned studies was carried out by Oreg (2006), who identified that different forms of resistance can indicate different types of antecedents, and thus would point to different measures to alleviate resistance. The investigation adopted the tridimensional attitude model for resistance to change and two 
main categories of context variables: anticipated change outcomes and change process.

In a study by Van Dam, Oreg et al. (2008), the change process was evaluated by three process variables; information, participation and trust in management, using a 7point Likert scale. One of the great findings in Oreg's study was that "Trust in management" is the only variable with significantly influences the affective, cognitive and behavior dimensions. It is also worth stressing that "Information" influenced the behavior and cognitive dimensions. Although it had been anticipated that there would be an inverted relation with cognitive dimension, that is, the more information given, the less resistance shown (based on the assumption that resistance is due to unfamiliarity with details of change), the opposite was found to be true. Oreg himself points out a possible explanation for it by stating that the relationship between information and resistance "would therefore appear to depend on the content, rather than on the mere existence of information. Furthermore, the manner in which the information is communicated is also likely to influence change acceptance." In fact, Oreg's thinking turns into provocative questioning not about investigating if information and participation are provided but in which ways they are provided to lighten resistance to change. As other authors alert, even when following classic prescriptions to deal with resistance by informing and allowing participation, there are authentic and feigned ways to do so.

The claim here is that participation will never work if it is treated as a device to get people to do what a change agent wants them to, as it could lead to deeper and even more disguised resistance to change. Genuine participation is based on respect, which comes from recognizing a real dependence on people's contributions. This would drive the agent to gather ideas and suggestions, not in a backhanded way to get compliance, but in a straightforward way to gather some good ideas and avoid some unnecessary mistakes. It would be possible to discuss, accept or reject ideas on their own merit.

Washington and Hacker (2005: 402) have already declared that "while many studies focus on the factors impacting change, the link between resistance and change 
has been only explored to a limited extent". In a similar vein, Van Dam and Oreg's (2008) previously claimed that, in the field of organizational change, "future research should expand the context characteristics studied". As an answer to those arguments, this research is an effort to address the still existing gap in the understanding of the mechanisms that allow resistance to change - understood as a communicative expression - to be embraced and positively managed. It seeks to clarify those mechanisms and contribute towards allowing practitioners to turn a so-called resistant into an acceptor or an advocate, and so that the desired change outcomes can be achieved.

Nonetheless, as the literature review on resistance to change has already indicated, the genuineness or authenticity of the participation process is configured as a relevant focus of investigation. Change would depend strongly on the ways information and participation are provided, not on the mere existence or amount of input, either given or requested (Lawrence, 1954; Powell and Posner, 1978; Stohl and Cheney, 2001; Lewis, 2006: 11). In other words, "it is not sufficient to offer the right structure or design in order to promote active participation" (Deetz, 1992). Thus, in order to contribute towards broadening the understanding of communication in relation to resistance to change, it seems valuable to turn the focus of future research on communication and change to the very nature of participation. As supported by other scholars:

"Participation changes the content and the form of the communicative event, although the extent to which it approaches authentic democratic dialogue remains an important question" (Stohl and Cheney, 2001: 359).

Those statements refer to numerous situations within organizations undergoing change processes in which participation is handled as an instrumental method to promote change compliance. What some scholars suggest is that those illegitimate participations may not contribute to minimizing resistance but, on the contrary, may make it subtle and harder to manage (Lawrence, 1954; Powell and Posner, 1978; Stohl and Cheney, 2001). 
By adopting a communication lens, the challenge of investigating the authenticity of participation could be better understood after considering Jabri et al's (2008) account that

"much of organizational change work around achieving consensus through participation in dialogue, remains quite monologic (one logic); even when a diversity of points of view interact in dialogue, the stress is placed upon achieving consensus, or in utilizing rhetorics of persuasion to arrive at common ground for all (to keep contentious point of view on the margin)...A supposedly polyphonic dialogue can remain monologic (one logic) and not achieve polylogical (multiple logic) aims" (Jabri, Adrian et al., 2008: 678-679).

As a consequence, it is possible to deduce that, on several occasions during participation activities designed to produce dialogic communication, this is not achieved and participants, although supposedly participating, are in fact engaged in a monologic communication through which they are at most allowed to voice their opinions without any real possibility of influencing the change process. Summing up, different participation processes may occur within a change implementation: what could be called dialogic participation (participants considered as authentic subjects) or monologic participation (where participants are treated as objects).

In order to operationalize the differences between these two proposed natures of participation, it is worth building up from the concept of dialogue, and one of the most incisive is offered by Heath et al:

"dialogue as the ability to state your perspectives, values, and desires while remaining open to the perspectives, values, and desires of others" (Heath, Pearce et al., 2006:341). 
In fact, the dialogue concept offers "a different perspective on participation: a perspective whereby one person's message joins with that of another and one person's meaning joins with that of another" (Jabri, Adrian et al., 2008). Moreover, a change that is led through dialogue is more likely "to take root because it is born at a point of contact among various consciousnesses" (Bakhtin, 1984:81).

"The difference resides essentially in understanding of the notion of participation "with the dialogic communication acknowledging "interpretive rights" in addition to acknowledging "voice", which can be encountered in monologic approaches". (Jabri, Adrian et al., 2008: 679)

Still in the direction of operationalizing those differences between the monologic and dialogic approaches, it is worth looking at a particular study. Frahm and Brown (2007) applied Kent and Taylors' (2002) five Principles of Dialogic Communication to Public Relations to an organizational change setting, and by means of comparisons between two organizations immersed in incremental change processes they established a starting point for further investigations. They studied the impact of change communication on change receptivity. The study employed a mixed methodology of surveys, participant observation, focus groups, and document analysis. Findings indicate that a shift from monologic to more dialogic communication occurred and improved receptivity to change.

Mutuality is the first principle, and it includes features of collaboration and spirit of mutual equality. It also means that participants in dialogue are viewed as persons and not as objects, or "targets of change". The exercise of power or superiority should be avoided in dialogue.

Propinquity is created by three features of dialogic relationships: "immediacy of presence," "temporal flow," and "engagement." Propinquity means that participants of a 
dialogue are engaged in the present, instead of after the decision-making. There is acknowledgement of past, present and future discussions. Propinquity is also related to a willing engagement in the process.

Empathy is about the environment of support and trust that must exist for dialogue to succeed. It embraces supportiveness, communal orientation and confirmation or acknowledgement. As already discussed and previously supported by Jabri et al (2008), Schein (2003) and Heath et al (2006), in dialogic communication participants demonstrate the "capacity to listen without anticipating, interfering, competing, refuting, or warping meanings into preconceived interpretations." (Frahm and Brown, 2003).

Risk is one of the key principles of dialogic communication, as it means that participants in a dialogic communication are able to recognize openly what they do not know and assume uncertainty as part of the process as well as the results. In such cases, the vulnerability of not having such control reveals itself as a position of strength rather than weakness, and then new meaning can be developed collectively.

Commitment can be understood as genuineness, commitment to conversation, and commitment to interpretations. Dialogue is honest and forthright. Sharing the same meanings or working toward common understandings is crucial to dialogic relationships, constantly fine-tuning language and trying to grasp the positions, beliefs, and values of others before their positions can be equitably evaluated.

Those principles were developed by Kent and Taylor (2002) after an extensive literature review in psychology, philosophy and communications. It is clear that a dialogic approach is not easily operationalized or reduced to a series of steps, but those principles consist of several coherent assumptions that can become the foundation needed to investigate the authenticity of a dialogic participation process. 
One of the challenges in promoting dialogic communication is to recognize the interpretive rights of others, something not easily achievable in one single interaction, and even more in one ongoing process. As Schein (2003) has already pointed out, the roots of dialogue lie in discovering our internal choice process regarding when to speak and what to say. The key to dialogue is to keep open to new interpretations, and this is deeply connected to resisting change. As the sensemaking process makes it clear, our own interpretations are implicit and our framings for reality are rarely conscious. It is by interacting with other participants that one ends up either validating or not one's own interpretations. All is well when interaction reveals alignment between interpretations. It is when responses vary from the expected that it is time to reconsider one's own interpretations, if there is an opening for doing so. However, if there is no acknowledgment of the other's right to interpret reality differently, the usual effect is to classify the response as not valid or to start a persuasion effort. Both interlocutors' reactions can be labeled as resistance, from each other's point of view.

Under dialogic communication and participation, unexpected reactions to a change process would not promptly be seen as resistance. In fact, responses are recognized as "expressions that are informed by what was said previously and what is happening currently", without the negative label of representing a threat to what has been agreed before. Really influencing change is later an essential indicator of a dialogic communication. When carried out under dialogic communication "change initiatives would be open to change as they were implemented." (Jabri, Adrian et al., 2008: 680).

That defines a proposed dimension to dialogic communication that was created by the author of this research, called Input Use: if through the input collected during communication effort the "change initiatives were open to change as they were implemented" (Jabri, Adrian et al. 2008: 680) 
If one considers that resistance does not come only from recipients but also from agents (Ford and Ford, 1995; Ford, Ford et al., 2008; Ford and Ford, 2009), and if agents do not truly receive and incorporate counter arguments into the content and process of change, then participation is not happening. In such cases, it is just voicing or considering participants as objects, which means it is monologic participation. Unfolding from the latest arguments, it is inferable that not all participation would minimize resistance as largely prescribed, but monologic participation could in fact lead to higher resistance.

Such understanding brings about the need to identify the implications in resistance to change of what could be identified as dialogic or monologic participation, and such a line of investigation would contribute to that line of research that explores the communicative nature of influences on change. Thus, it would be simultaneously strongly coherent with the logic underpinning a participatory approach (Russ, 2008) to constructivist ontology. Besides, as other scholars have already alerted, for practitioners to be able to make good use of advice on involving employees when dealing with resistance to change, they need to know how employees react to the different nature through which such participation can be promoted (Seibold and Shea, 2001; Lewis, 2006). This study is one step towards closing this gap in current research.

\section{Methodology}

Several studies of communication and Resistance to Change - RTC adopted an instrumental approach that focuses mainly on the informational nature of communication. Communication nature have been studied with different emphasis, for example on structure for participation, levels of hierarchy of the audiences involved, or the opportunity to voice opinions during different stages of change implementation. Taking into consideration that different natures of communication (monologic and dialogic) co-exist during change processes, "different forms of communication and change may simultaneously exist within organizations" (Waterhouse and Lewis 2004: 355), the objectives for the case study were: 
- Develop a scale and instrumental grid to explore communication nature dimensions and identify the predominant nature of the described communication activities, if monologic or dialogic;

- Identify levels of resistance to change and its dimensions, concomitant to those same change prevalent communication natures;

- Explore what are the contributions or implications of internal monologic or dialogic communication natures to resistance to change and towards resistance to change and ultimately to implementation of radical change.

This case study has a holistic approach, once its unit of analysis is the corporatecommunication strategy at organizational level and some units of observation (organization, change leaders, employees, and communication experts) would be analyzed as they can offer opportunities to elicit the relation between communication nature and RTC dimensions (Yin 2010:74,76).

\section{Operational Descriptions}

In this research there are two main propositions and two main operational descriptions to be clarified.

Corporate communication nature may vary during an organizational change context period of time. The proposal is to evaluate nature of communication according to the observance of the elements of dialogic communication, which are Mutuality, Propinquity, Risk, Empathy, Commitment, Input Use. The presence of those principles in an organizational communication would indicate the occurrence of a dialogic communication, although low levels of some of the principles would not lead to monologic, but to a weaker dialogic communication. The lack of all principles would be the characterization of monologic communication.

To evaluate the perception of Organizational Resistance to Change according to three dimensions (cognitive, affective and behavioural), the scale developed by Oreg (2006). was adapted to be collective oriented. It will work as a frame about how 
individuals perceived the resistance to change as an expression by others, the organizationas whole.

The first stage, a qualitative one, looked for to characterize the corporate communication activities related to change in each organization, by collecting data through observation, semi-structured interviews and questionnaires with the main change leaders and communication managers. Also it was collected material for document analysis and made direct observation to check the data. Such choices were made according to proposals made by several authors (Minayo, Assis et al. 2005; Yin 2005) about how appropriate multiple sources of evidence can be towards a more encompassing data collection process. Thus, triangulation was made possible and, consequently, the research intend to be more successful from the point of view of questionings as to its legitimacy (Eisenhardt and Graebner 2007).

As a complement for the managers' view of the change and change communication nature, it were carried out group discussions, to elicit employees understanding of what type of change was occurring, how they felt about it and their beliefs about how communication of change was conducted.

\section{FINDINGS AND DISCUSSION- PILOT CASE STUDY -Organization X}

\section{Organization Description}

Organization $X$ is a company froma large multinational group nowadays, from now on referred to as The Group. The company is amarket leader in Generic Medicines in Brazil since 2002, with a portfolio of more than 150products. In 2008, the company sales amounted to $\mathrm{R} \$ 458$ million. Organization $X$ was the third largest pharmaceutical company in Brazil. Their good performance is due mainly to the generic medicines market, where the company achieved $55 \%$ growth versus $38 \%$ of the market during 2010.

The Group is a world leader in the pharmaceutical industry and research, developing and publicizing therapeutic solutions to improve the lives of people. Present 
in over 100 countries, The Group is a global company, diversified in health care. With over 100,000 employees, The Group is the world's one of thelargest pharmaceutical companies in the world, and is leading the so-called emerging countries. The vaccines business of the group, is a world leader in the production and marketing of human vaccines. The Group is an open-capital enterprise with shares traded in the Paris and New York stock exchanges.

The announcement of the deal was done in April, 2009 inthe, headquarters of the conglomerate. The acquisition strengthens market strategies of two groups: betting on emerging markets - and in Brazil and Latin America in particular - and growth in generic drug sales.

\section{Change Context}

Some months before the acquisition, Organization $\mathrm{X}$ was facing a very difficult financial situation. Although extremely respected by its partners and customers, the organization had accumulated a considerable dependence on bank money. Suppliers were already concerned and the employees were in a climate of uncertainty every month, wondering about being paid, being laid off or keeping the job longer. The rumors prevailing on the market over the previous year were already about the possibility of selling the company.

When the acquisition of Organization $X$ byTheGroup was announced in April, 9th of 2009, the main feeling wasof relief. Both the employees and the board were satisfied with the perspectives that such a strong financial support, coming from a respected pharmaceutical global group, would represent for the short and long terms.

\section{$\underline{\text { Communication }}$}

There were formal (written) and face communications announcing the acquisition. A letter signed by Organization X's president at that time, addressed to the employees, reinforced the ending of a hard time and the beginning of a new stage for the company. 
The entire days of 08 and 09 of April were dedicated by the president to personally explain the immediate consequences and clarify the most common doubts that the acquisition would generate for the employees. Face meetings in all shifts in both plants were organized so the conversation could address items such as if it was partial or complete acquisition, if planned investment would change, if the board would change, if benefits for employees would change, etc.

An internal face to face communication plan was put together by HR and the president himself, following an established tradition of openness about the main facts involving the organization. The meeting was formatted to start with a speech, give some answers to the questions that were anticipated in the plan and open to questions from the audience. That is revealing about the nature of communication for this moment; open, direct and frank.

In the end of April 2009 already there were a group of people from The Group working on Organization X's sites. The first team was massively formed of finance people, including a new Finance Director, exactly because of the cash problems the company was facing, and also constituted by Human Resource and Business Support directors.

According to the interviewees the first three directors that were invited to integrate Organization X, coming from The Group, they all received a very clear briefing from TheGroup'sPresident in Brazil, about their roles and the way the integration should be carried out:

"We received three messages: "Openthedrawers,look ateverythingandfind outhowthe business works", the firstpoint. Secondpoint, "preserve the culture, though youarefrom the Group,preserve Organization X's culturebecause it hasavery strong thingthereandthat has tobepreserved".And thethird point, "protect the people."' (Interviewee G) 
Those clear instructions about keeping the culture were in consonance with the objective of maintaining separate business models and structures, while integrating back office operations. Generic Medicines was not yet part of The Groupexpertise, although it already produced in Suzano / SP a large portfolio of branded drugs, vaccines and OTC (Over-the counter, that is, medicines that may be sold directly to a consumer without a prescription from a healthcare professional).Organization X's expertise and success in generic market was to be respected, while at the same time, combined to The Group management style.

The preservation of the culture was explicitly requested in several stages, not only in the moment right after the acquisition, but also later, in July/2010, more than a year later, this was again solicited for the new General Director, that was replacing the former Organization X's President:

The process of integration has been considered well succeeded till now, by majority of testimonies collected and change has its meaning connected to different decision processes, levels of control and formal standard procedures. The process is modifying while The Group is learning about the generic business model. It is interesting to notice also that together with the change in management system, there has been also a perception about the occurrence of a cultural change. What could be interpreted as a confronting result in relation to the guidelines previously posed, about preserving the culture, seems to be clarified by one of the respondents that framed that within culture, the identity is what was really meant to be conserved.

Looking further and deepening the meaning of change for Organization X's interviewees, stands out the differences in decision process and power distribution two years after integration. Firstly highly concentrated in the President and VP's now it is more decentralized. This new characteristic of decentralization of decision making and power is also richly described inside the organization by the word transversality, largely used at the time of data collection. 
In terms of the quality and amount of information available concerning the change there is a general understanding that both quality and quantity were adequate. Quality of information seemed to be well evaluated too, but with space to develop through detailing and timing.

The speed of change, new initiatives, adjustments and projects, as well as the great amount of procedures, patterns and policies to be aware of, are the causes for the general perception about the needed care in terms of quality of information. In fact, no new vehicles of communication were created after acquisition, and the existing ones were preserved.

It seems that an informal and open internal climate helped to keep the flow of information during the change process.

The general evaluation of Organization X's communication is that it had since before the acquisition, a highly fertile starting point, where of one assuming that didn't know something did not mean a power loss (Risk dimension) and where it was common language fine-tuning and trying to grasp others positions (Commitment). As can be observed in Graphic 01 these two dimensions were the higher ones at the time of acquisition (DA ACQ), according to respondents evaluation. That is coherent to the descriptions former presented about the internal climate and the decision making process. As also can be seen below in Graphic 01, Propinquity, Mutuality, Empathy and Input were in this order, not very present right after the acquisition, about two years before the first field data collection, compared to Risk and Commitment that were already at good levels and had some variation, but remained so six months before data collection (DA SMA) and also at March 2011 (DA MA11).

Previous to the acquisition, vice-presidents for instance would usually took decisions separately and announce them to the rest of the organization, characterizing that participants of a dialogue were engaged after the decision-making (Propinquity), and that was gradually altered by the new management model, reflecting later in the growth of this item evaluation. The great charismatic style of the former president is an 
iconic example of how Mutuality was different under his leadership, in terms of his of unquestioned power. As previously stated by respondents, not that it was generating a bad climate, on the contrary, people were very pleased to count on a fair leader, visionary and excellent communicator.

In terms of the environment of support and trust that must exist for dialogue to succeed (Empathy) it is clear this was largely facilitated by the informal atmosphere that already existed in Organization $\mathrm{X}$ before the acquisition. Right after the acquisition this was not higher due to the unknown interlocutors and the uncertainty that prevailed in the context.The same can explain the Input level, that is, change initiatives were open to change as they were implemented, as the following example:

"...In construction stage we were involving more and more people. I wished they could talk. First I needed to know the background; I needed to master the sales managers and needed to measure the reactions $(C)$. Then it was deliberate and left them talk. But I always did interventions because, "this point has created a controversy here ... You remember the date of such meeting, we have a record, and look what you have told us there ... Never mind, if we come to the conclusion that this should be changed it will be changed ...(P). And what I want to make clear is that this item was built like that by your observations $(M)$.If we come to the conclusion that today it is better to change, no problem. We change" $(P)$. (Interviewee G).

In terms of the Resistance to Change, the largest fall observed was in affective dimension, meaning feelings of fear, anger or anxiety were the stronger component in resistance at the beginning, but not anymore as reported by the participants of the research.

As explained earlier, there was an understanding concerning the acquisition, it was comprehended since the first stages of the change process, as a good solution for the financial instability the company was immersed. Cognitive is still the weakest dimension of resistance to change for the respondents. People know they have to 
adjust as now they belong to a multinational corporation, with global standards, they understand there is more work to do, more procedures to follow.

There is also an understanding of improvements after integration, in terms of management as well in terms of having global experiences, exposition and professional opportunities of growth.

There are evidences of self-consciousness about the process and justification about how new procedures and activities need to beadjusted. That reveals The Group is respected by its management know-how, while Organization $X$ is respected by its generic marketknow-how.

Interestingly, behavioral dimension of resistance to change according to the participants of the research, has also fallen down, but not in the same scale compared to the other dimensions. One possible explanation is the fact that nowadays there is institutional support to act or express the intention to act in response to the change (e.g., complaining about the change, trying to convince others that the change is bad), and so people are encouraged to act. This is expected in regular meetings as a contribution to shaping the change itself, through the transversality practice.

Another complementary explanation is that as many changes are related to establishing patterns and so called bureaucratic procedures, and as generic market is so "nervous" and fast, employees really need to find ways to proceed and overcome what is perceived sometimes as bureaucracy if they want to keep the speed and innovation that the strategic relationship positioning adopted by Organization $\mathrm{X}$ really requires. In other words, finding ways to adjust patterns, surmount procedures and provoke ruptures is what is expected and allowed to do (dialogic communication), but that takes time, and becomes a matter to this business how get to quicker solutions without injuring organizational practices of transversality and dialogue. 


\section{Conclusions}

By overlapping the data about communication characterization and resistance to change characterization in Organization $X$, respecting the limits of these analysis to respondent views, during these two first years after acquisition, it can be noticed a direct opposite movement. While communication was becoming highly dialogic the resistance to change was descending. As it is not derived from statistical analysis, from a generalizable sample, it cannot be concluded that it is a cause-effect relationship. But, from a qualitative angle, of expressing how both constructs are related it is quite valuable.

As can be observed in Graphic 01, all dimensions of Dialogic Communication are going up and all dimensions of Resistance to change are going down over the time. Obviously, the period average indicator of both Communication and RTC follow the same opposite path. It is clearly observed in Graphic 02, where the averages are presented separately from their components.

\section{Graphic01}

Communication and Resistance to Change Evolution

Organization X

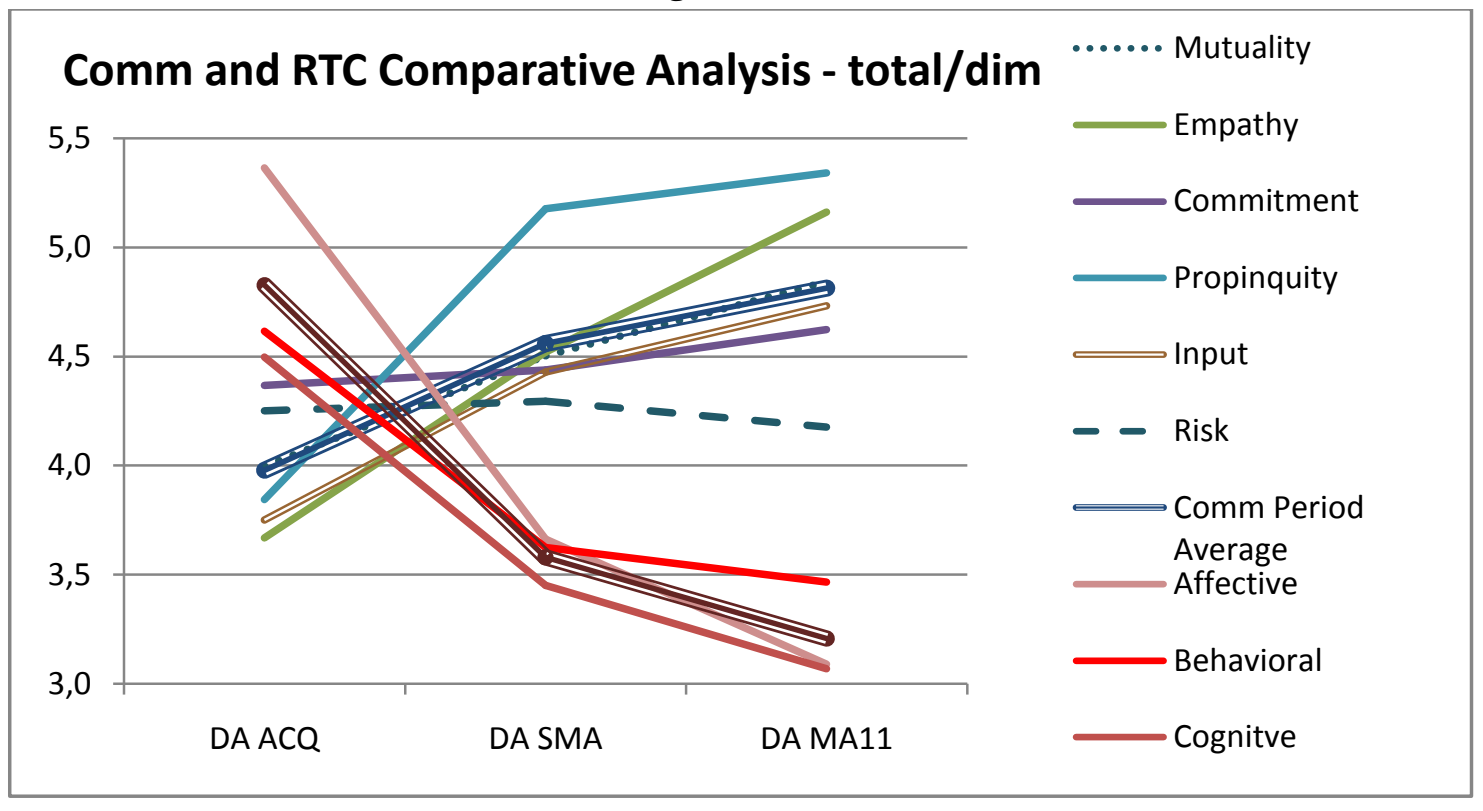

Graphic02 
Communication and Resistance to Change Average Evolution

Organization X

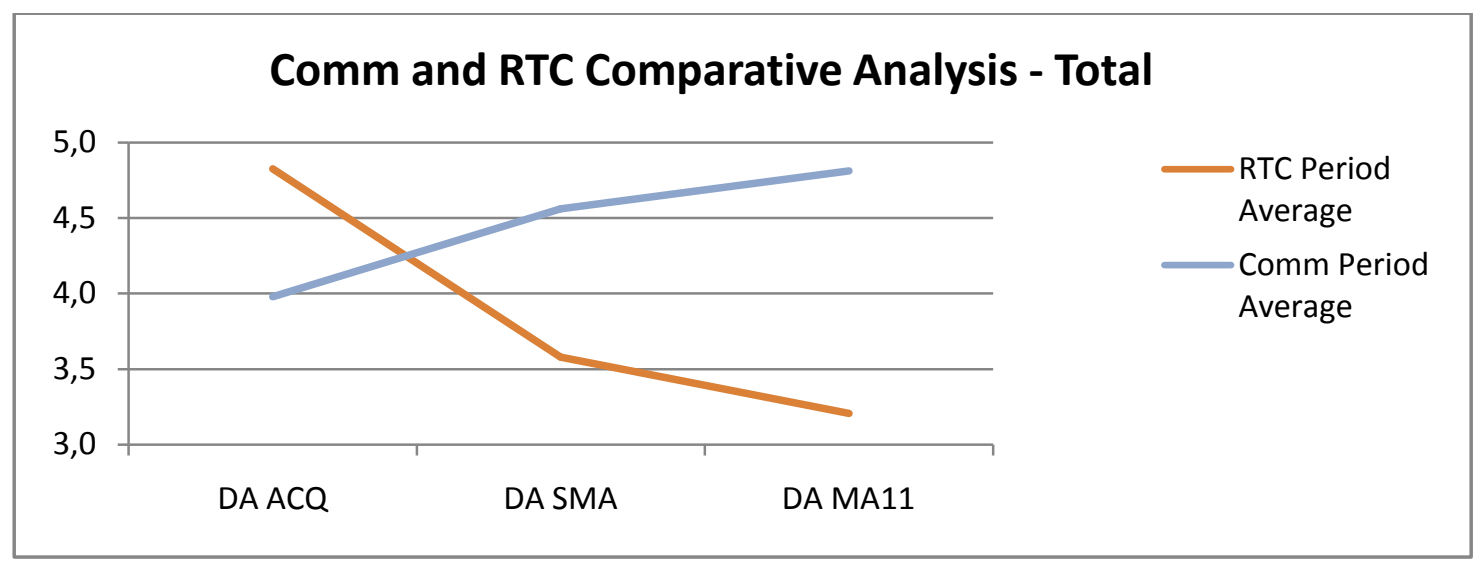

According to Organization X's General Director, communication was a key aspect to endorse a good change process, by means of maintaining identity symbols and promoting dialogic opportunities:

"We're investing a lot in communication. I think in a process of Change Management may not have anything more important to calm people down than good communication. You do not communicate it's a way to communicate. You do not say anything, you're saying something to the employee. So if you do not say anything, he already knows, somehow you're telling him what he wants to know. So we try and we have to be nearby. And better than to hear in the hallways or musing assumptions, we have to showclearly and talk about what is happening. .... We kept some symbols of the company, some things that were highly valued by the company ... And people actually lived this, how cool is Organization X. And we kept it, as well as the famous June Festival of the company, ranging families, wives, husbands and children, a symbol ofOrganization $X$, and we kept going and continue to hold. So we try to respect the elements, company logos, things really valued by the company and try to introduce some more. ... think this is the most important, I think we have to communicate, has to explain why we're going in one direction and all have to believe we are headed in the right direction. And at least give the space to people talk, "look, I do not believe, or, I want to give a suggestion".(Organization $X$ General Director) 
It is clear also, that it's not only a communication department task, but an HR challenge, in the sense that communication nature is institutionally shaped and also individually shaped.

This constant evaluation and care about the change process seems to be ongoing, as many respondents highlighted they are still not mastering the identification of when there are really dialogic opportunities and where it is just monologic communication. They're still not comfortable with the explicit drive of being dialogic and the paradoxical need to conform - as some monologic communication (from standard procedures and high control) is naturally expected to exist after acquisition. It is worth noticing that monologic is named as information, strongly adherent to the concept earlier treated in the literature review.

At Organization $X$ it seems the change process is perceived as being successful, with what could be considered good levels of resistance to change, due to the very explicit endorsement of dialogic communications.

"The success it's there, we're selling more and more. .... So this is the first success, we're selling a lot. The second, I think is the way it was done. The decisions were postponed(P), and some sort of pushing sometimes happened in one area or another, but the collegial decisions(M), even if you did not agree on the whole decision, you have signed below, and then you took for your group. And over time this was proving to be the best decision. And this helped to gee, really helped, I think is the greatest success. And the other was the freedom we had, because while we have here faced some walls, on the other side had allThe Group employees"they are doing something that cannot be done in the group". And we found a middle path formula(I) ... do not ask me how! It was coming up, things were coming up and we were addressing the extent possible. And then I think it was the coolest thing of all. And I feel very happy, very happy."(Interviewee G).

By indicating not only the relation among dialogic communication and resistance to change, but identifying the dimensions under those constructs that explain them, it is 
possible for academia and organizational worlds to keep developing better understanding and conducting of change processes.

A relevant implication was identified for practitioners within organizational change process, mainly in terms of the guidance to organizational leaders. Usually rests on the leadership capability of each manager to select and implement a communication style with his/her team. But after comprehending that mutuality, risk, input, propinquity, empathy and commitment are influential to resistance to change, the organizational leaders can design the change implementation to attend those requisites. Also, after each main step within the change management process, a communication guideline can be created and shared to enlighten its deployment over different areas and hierarchical levels.

Future research can explore the existing relation between each dimension of both constructs and also apply the same methodology in different contexts, as only one size of organizations, different environmental causes for changes and other variables of the change process.

Another way of advancing in research would be to conduct only the quantitative constituent of the methodology proposed for this case study, in an statistic relevant sample of organizations under radical change process, aiming to establish a causal mathematic relation between dialogic communication and resistance to change. 


\section{References}

Burnes, B. and H. James (1995). "Culture, cognitive dissonance and the management of change." International Journal of Operations \& Production Management 15(8): 14.

Daft, R. L. and K. E. Weick (1984). "Toward a Model of Organizations as Interpretation Systems." Academy of Management Review 9(2): 284-295.

Eisenhardt, K. M. (1989). "Building Theories from Case Study Research." Academy of Management Review 14(4): 532-550.

Eisenhardt, K. M. (1989). "MAKING FAST STRATEGIC DECISIONS IN HIGHVELOCITY ENVIRONMENTS." Academy of Management Journal 32(3): 543-576.

Eisenhardt, K. M. and M. E. Graebner (2007). "THEORY BUILDING FROM CASES: OPPORTUNITIES AND CHALLENGES." Academy of Management Journal 50(1): 2532.

Ford, J. D., L. W. Ford, et al. (2008). "RESISTANCE TO CHANGE: THE REST OF THE STORY." Academy of Management Review 33(2): 362-377.

Frahm, J. A. and K. A. Brown (2003). "Organizational change communication: Lessons from public relations communication strategies."

Greenwood, R. and C. R. Hinnings (1996). "Understanding Radical Organizational Change: Bringing Together the Old and the New Institutionalism." Academy of Management Review 21(4): 1022-1054.

Heath, R. L., W. B. Pearce, et al. (2006). "The Processes of Dialogue: Participation and Legitimation." Management Communication Quarterly 19.

Jabri, M., A. D. Adrian, et al. (2008). "Reconsidering the role of conversations in change communication A contribution based on Bakhtin." Journal of Organizational Change Management 21(6): 667-685.

Lawrence, P. R. (1954). "How to Deal with Resistance to Change." Harvard Business Review 32(3): 49-57.

Lewis, L. K. (2006). "Employee Perspectives on Implementation Communication as Predictors of Perceptions of Success and Resistance." Western Journal of Communication 70(1): 23-46.

Minayo, M. C. d. S., S. G. d. Assis, et al. (2005). Avaliação por triangulação de métodos: abordagem de programas sociais. Rio de Janeiro, Fiocruz. 
Palmer, I. and R. Dunford (2008). "Organizational Change and the Importance of Embedded Assumptions." British Journal of Management 19: S20-S32.

Powell, G. and B. Z. Posner (1978). "Resistance to Change Reconsidered: Implications for Managers." Human Resource Management 17(1): 29-34.

Schein, E. H. (2003). "On Dialogue, Culture, and Organizational Learning." Reflections 4(4): 27-38.

Stake, R. E. (2000). Case studies. Handbook of qualitative research. N. K. L. Denzin, Y. S. London, Sage: $435-454$.

Stohl, C. and G. Cheney (2001). "Participatory Processes/Paradoxical Practices." Management Communication Quarterly 14(3): 349.

Taylor, J. R. and E. J. Van Every (2000). The emergent organization: Communication as its site and surface. Mahwah, NJ US, Lawrence Erlbaum Associates Publishers.

Waterhouse, J. and D. Lewis (2004). "Communicating culture change." Public Management Review 6(3): 353-376.

Weick, K., Ed. (1979). The social psychology of organizing, Reading, Mass.: AddisonWesley.

Weick, K. E. and R. E. Quinn (1999). "Organizational change and development." Annual Review of Psychology 50(1): 361.

Yin, R. K., Ed. (2005). Case study research: desing and methods. Applied Social Research Methods Series. Los Angeles, Sage Publications.

Yin, R. K., Ed. (2010). Case Study Research: Design and Methods. Porto Alegre, Bookman. 